Commun. Fac. Sci. Univ. Ank. Ser. A1 Math. Stat.

Volume 68, Number 2, Pages 1950-1958(2019)

DOI: $10.31801 /$ cfsuasmas.000000

ISSN 1303-5991 E-ISSN 2618-6470

http://communications.science.ankara.edu.tr/index.php?series=A1

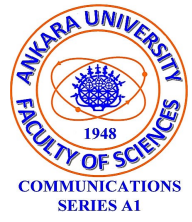

\title{
LOXODROMES ON HELICOIDAL SURFACES AND TUBES WITH VARIABLE RADIUS IN $\mathbb{E}^{4}$
}

\author{
MURAT BABAARSLAN
}

\begin{abstract}
In this paper, we generalize the equations of loxodromes on helicoidal surfaces and canal surfaces in $\mathbb{E}^{3}$ to the case of 4 -dimension $\left(\mathbb{E}^{4}\right)$. Also we give some examples via Mathematica.
\end{abstract}

\section{INTRODUCTION}

A curve which cuts all meridians at a constant angle on the Earth's surface is called as loxodrome. Loxodromes don't need a change of course and thus, they are usually used in navigation. Noble [1] investigated the equations of loxodromes on the rotational surfaces in Euclidean 3-space $\mathbb{E}^{3}$. The orbit of a plane curve under a screw motion is called as helicoidal surface and it is a natural generalization of rotational surface. The equations of loxodromes on helicoidal surfaces in $\mathbb{E}^{3}$ were found by Babaarslan and Yayli $[3]$.

Another generalization of rotational surfaces is canal surfaces and they are defined as envelope of a family spheres whose trajectory of centers lie on a space curve. When the radius of spheres is constant, the canal surfaces reduce to tubes with constant radius 12. Also, if the centers of spheres lie on a straight line, then the canal surface is a rotational surface [9. For example, the sphere is a special canal surface whose axis is a straight line. The differential equations of the loxodromes on canal surfaces in $\mathbb{E}^{3}$ were given by Babaarslan 4 .

Rotational surfaces in Euclidean 4 -space $\mathbb{E}^{4}$ was first introduced by Moore [10]. After that, a lot of authors studied on rotational surfaces in $\mathbb{E}^{4}$ (see [13, [14, [1, [2, 6]).

The parametrization of tube with variable radius in $\mathbb{E}^{4}$ was given by Gal and Pal 9. Also, the definition and parametrization of helicoidal surface in $\mathbb{E}^{4}$ were given by Hieu and Thang $[8]$.

Received by the editors: August 27, 2018, Accepted: April 18, 2019.

2010 Mathematics Subject Classification. 53B25.

Key words and phrases. Loxodrome, helicoidal surface, tube with variable radius, Euclidean 4-space.

(C)2019 Ankara University Communications Faculty of Sciences University of Ankara-Series A1 Mathematics and Statistics 
In this paper, we generalize the equations of loxodromes on helicoidal surfaces and canal surfaces in $\mathbb{E}^{3}$ to the case of 4 -dimension $\left(\mathbb{E}^{4}\right)$. Also, we give some examples by using Mathematica computer programme.

\section{Preliminaries}

In this section, we recall some important notions and also give some properties of curves and surfaces in $\mathbb{E}^{4}$.

Let $x=\left(x_{1}, x_{2}, x_{3}, x_{4}\right)$ and $y=\left(y_{1}, y_{2}, y_{3}, y_{4}\right)$ be vectors in $\mathbb{E}^{4}$. Then, the inner product of them is given by

$$
\langle x, y\rangle=x_{1} y_{1}+x_{2} y_{2}+x_{3} y_{3}+x_{4} y_{4} .
$$

The norm (length) of a vector $x \in \mathbb{E}^{4}$ is defined by $\|x\|=\sqrt{\langle x, x\rangle}$ and the vector is called as a unit vector if $\|x\|=1$.

Also, the angle $\theta$ between $x$ and $y$ is given by

$$
\cos \theta=\frac{\langle x, y\rangle}{\|x\|\|y\|}
$$

where $0<\theta<\pi$.

Let $\beta: I \subset \mathbb{R} \rightarrow \mathbb{E}^{4}$ be a regular curve in $\mathbb{E}^{4}$. The arc-length of $\beta$ between $t_{0}$ and $t$ is

$$
s(t)=\int_{t_{0}}^{t}\left\|\beta^{\prime}(t)\right\| d t .
$$

Then, the parameter $s \in J \subset \mathbb{R}$ is determined such as $\left\|\beta^{\prime}(s)\right\|=1$. Thus, $\beta$ is called a unit speed curve if $\left\|\beta^{\prime}(s)\right\|=1$.

Now, we give the definitions and parametrizations of rotational surfaces and helicoidal surfaces in $\mathbb{E}^{4}$.

Let $\beta: I \subset \mathbb{R} \rightarrow \Pi$ be a smooth curve in a hyperplane $\Pi \subset \mathbb{E}^{4}$ and $P$ be a 2plane line in $\Pi$. If the profile curve $\beta$ is rotated about $P$, then the resulting surface is rotational surface in $\mathbb{E}^{4}$. Similarly, let us assume that when $\beta$ rotates about $P$, it simultaneously translates along a line $l$ parallel to $P$ so that the speed of the translation is proportional to the speed of rotation. Then, the resulting surface is a helicoidal surface in $\mathbb{E}^{4}$ (see [8]).

Let $x, y, z, w$ be the coordinates in $\mathbb{E}^{4}$. We assume that $\Pi$ is $x z w$-hyperplane, $P$ is $z w$-plane and $l$ is parallel to the $z$-axis. Then, the rotation which leaves the plane $P$ invariant is given by the following rotational matrix

$$
\left[\begin{array}{cccc}
\cos v & -\sin v & 0 & 0 \\
\sin v & \cos v & 0 & 0 \\
0 & 0 & 1 & 0 \\
0 & 0 & 0 & 1
\end{array}\right], 0 \leq v<2 \pi
$$

77. 
We consider the profile curve $\beta(u)=(f(u), 0, g(u), h(u))$ in $\Pi$, where $u \in I \subset \mathbb{R}$ and $f(u)>0$. Then, the parametrization of the helicoidal surface $M$ is

$$
M(u, v)=\left[\begin{array}{cccc}
\cos v & -\sin v & 0 & 0 \\
\sin v & \cos v & 0 & 0 \\
0 & 0 & 1 & 0 \\
0 & 0 & 0 & 1
\end{array}\right]\left[\begin{array}{c}
f(u) \\
0 \\
g(u) \\
h(u)
\end{array}\right]+\lambda v\left[\begin{array}{l}
0 \\
0 \\
1 \\
0
\end{array}\right]
$$

so

$$
M(u, v)=(f(u) \cos v, f(u) \sin v, g(u)+\lambda v, h(u)),
$$

where $\lambda>0$. When $g$ is a constant function, the helicoidal surface is called the right helicoidal surface. When $\lambda=0$, the helicoidal surfaces reduce to rotational surfaces in $\mathbb{E}^{4}$. Also, when $h$ is a constant function, the surface is just a helicoidal surface in $\mathbb{E}^{3} 8$.

Also, we give the parametrizations of tubes with variable radius in $\mathbb{E}^{4}$.

We consider the spine curve $\beta(u)=(f(u), g(u), h(u), 0)$, where $u \in J \subset \mathbb{R}$, that is $\beta$ is parametrized by arc-length. Then, the Frenet formulae is given by

$$
\begin{gathered}
\beta^{\prime}(u)=e_{1}(u), \\
e_{1}{ }^{\prime}(u)=\kappa(u) e_{2}(u), \\
e_{2}{ }^{\prime}(u)=-\kappa(u) e_{1}(u)+\tau(u) e_{3}(u), \\
e_{3}{ }^{\prime}(u)=-\tau(u) e_{2}(u), \\
e_{4}^{\prime}(u)=0,
\end{gathered}
$$

where $\left\{e_{1}(u), e_{2}(u), e_{3}(u), e_{4}(u)\right\}$ is Frenet orthonormal basis of $\beta, \kappa(u)$ and $\tau(u)$ are the curvatures of $\beta$.

Then, the parametrization of tube with variable radius $C$ is

$$
C(u, v)=\beta(u)+r(u)\left(e_{3}(u) \cos v+e_{4}(u) \sin v\right) .
$$

$([9],[5])$

\section{The Equations of Loxodromes on Helicoidal Surfaces}

In this section, we find the equations of loxodromes on the helicoidal surfaces as well as rotational surfaces in $\mathbb{E}^{4}$. Also, we give an example to strengthen our main results.

Definition 1. A curve on a helicoidal surface in $\mathbb{E}^{4}$ is called as a loxodrome if the curve cuts all meridians at a constant angle on the helicoidal surface.

Let us consider the helicoidal surface $M$ which is given by Eq. (5). To simplify the calculations, we assume that $\beta$ is parametrized by arc-length, i.e., $f^{\prime 2}(u)+$ $g^{\prime 2}(u)+h^{\prime 2}(u)=1$ for all $u \in J \subset \mathbb{R}$. 
The tangent plane to $M$ at a point $p=M(u, v)$ is $\operatorname{span}\left\{M_{u}, M_{v}\right\}$. A direct computation yields

$M_{u}=\left(f^{\prime}(u) \cos v, f^{\prime}(u) \sin v, g^{\prime}(u), h^{\prime}(u)\right)$ and $M_{v}=(-f(u) \sin v, f(u) \cos v, \lambda, 0)$.

By using these equations, the coefficients of first fundamental form of $M$ are

$$
E=\left\langle M_{u}, M_{u}\right\rangle=1, F=\left\langle M_{u}, M_{v}\right\rangle=\lambda g^{\prime}(u) \text { and } G=\left\langle M_{v}, M_{v}\right\rangle=f^{2}(u)+\lambda^{2} \text {. }
$$

Assume that $E G-F^{2}=\lambda^{2}\left(1-g^{\prime 2}\right)+f^{2}>0$, that is, $M$ is regular.

The first fundamental form of $M$ is

$$
\left.d s^{2}=d u^{2}+2 \lambda g^{\prime 2}(u)+\lambda^{2}\right) d v^{2} .
$$

Also, the arc-length of any curve on $M$ between $u_{1}$ and $u_{2}$ is given by

$$
s=\left|\int_{u_{1}}^{u_{2}} \sqrt{1+2 \lambda g^{\prime}(u) \frac{d v}{d u}+\left(f^{2}(u)+\lambda^{2}\right)\left(\frac{d v}{d u}\right)^{2}} d u\right| .
$$

Suppose that $\alpha(t)$ is a curve on $M$. Then, we can write $\alpha(t)=M(u(t), v(t))$. With respect to the local base $\left\{M_{u}, M_{v}\right\}$, the vector $\alpha^{\prime}(t)$ has the coordinates $\left(u^{\prime}, v^{\prime}\right)$ and the vector $M_{u}$ has the coordinates $(1,0)$. At the point $p=M(u, v)$, where the loxodrome cuts the meridian at a constant angle $\theta$, we get

$$
\cos \theta=\frac{d u+\lambda g^{\prime}(u) d v}{\sqrt{d u^{2}+2 \lambda g^{\prime 2}(u) d u d v+\left(f^{2}(u)+\lambda^{2}\right) d v^{2}}} .
$$

Then, Eq. (11) can be expressed in the form:

$$
\left(\cos ^{2} \theta\left(f^{2}(u)+\lambda^{2}\right)-\lambda^{2} g^{\prime 2}(u)\right)\left(\frac{d v}{d u}\right)^{2}-2 \lambda \sin ^{2} \theta g^{\prime}(u) \frac{d v}{d u}=\sin ^{2} \theta .
$$

This is differential equation of the loxodromes on the helicoidal surfaces in $\mathbb{E}^{4}$. Thus, the general solution of Eq. (12) becomes

$$
v=v(u)=\int_{u_{0}}^{u} \frac{2 \lambda \sin ^{2} \theta g^{\prime}(u)+\varepsilon \sqrt{\sin ^{2} 2 \theta\left(f^{2}(u)-\lambda^{2}\left(g^{\prime 2}(u)-1\right)\right)}}{2 \cos ^{2} \theta\left(f^{2}(u)+\lambda^{2}\right)-2 \lambda^{2} g^{\prime 2}(u)} d u,
$$

where $\varepsilon$ is plus or minus.

Then, we can give the following theorem.

Theorem 1. The loxodromes on the helicoidal surfaces in $\mathbb{E}^{4}$ are

$$
\alpha(u)=(f(u) \cos v(u), f(u) \sin v(u), g(u)+\lambda v(u), h(u)),
$$

where $v(u)$ is given by Eq. (13).

When $\lambda=0$ in Eq. (13), we find the following general solution of differential equation of the loxodromes on the rotational surfaces in $\mathbb{E}^{4}$

$$
v=v(u)=\varepsilon \tan \theta \int_{u_{0}}^{u} \frac{d u}{f(u)} .
$$


Thus, we have

Theorem 2. The loxodromes on the rotational surfaces in $\mathbb{E}^{4}$ are

$$
\gamma(u)=(f(u) \cos v(u), f(u) \sin v(u), g(u), h(u)),
$$

where $v(u)=\varepsilon \tan \theta \int_{u_{0}}^{u} \frac{d u}{f(u)}$.

If $g$ is a constant function, then the arc-length of loxodrome on the right helicoidal surface in $\mathbb{E}^{4}$ is given by

$$
s=\left|\frac{u_{2}-u_{1}}{\cos \theta}\right| .
$$

Similarly, the arc-length of the loxodrome on the rotational surface in $\mathbb{E}^{4}$ coincides with Eq. (15).

Now, we give the following example.

Example 1. Let us consider the profile curve $\beta(u)=\left(\cos \frac{u}{2}, 0, \sin \frac{u}{2}, \frac{\sqrt{3}}{2} u\right)$. If we take $\lambda=1, \theta=\frac{\pi}{2}$ and $\varepsilon=1$, then we have the following helicoidal surface:

$$
M(u, v)=\left(\cos \frac{u}{2} \cos v, \cos \frac{u}{2} \sin v, \sin \frac{u}{2}+v, \frac{\sqrt{3}}{2} u\right) .
$$

By using Eq. (13) and taking $u_{0}=0$, we get $v(u)=4 \ln \left|\frac{1-\tan \frac{u}{4}}{1+\tan \frac{u}{4}}\right|$. Taking $u \in(-2,2)$, we have $v \in(-4.9048,4.9048)$.

Then, the loxodrome is

$$
\alpha(u)=\left(\cos \frac{u}{2} \cos v(u), \cos \frac{u}{2} \sin v(u), \sin \frac{u}{2}+v(u), \frac{\sqrt{3}}{2} u\right)
$$

where $v(u)=4 \ln \left|\frac{1-\tan \frac{u}{4}}{1+\tan \frac{u}{4}}\right|$. Also, by using Eq. (10), the arc-length of the loxodrome is approximately equal to 12.0528.

Let us plot the graphs of the projections of helicoidal surface, loxodrome and meridian $(v=0)$ in $\mathbb{E}^{3}$ to see what they look like in $\mathbb{E}^{3}$ by using Mathematica plotting command

ParametricPlot3D $[\{x(u, v), y(u, v), z(u, v)+w(u, v)\},\{u, a, b\},\{v, c, d\}] ;$

\section{The Equations of Loxodromes on Tubes with Variable Radius}

In this section, we find the differential equations of loxodromes on the tubes with variable radius in $\mathbb{E}^{4}$. Also, we give an example to strengthen our main results.

Definition 2. A curve on a tube with variable radius in $\mathbb{E}^{4}$ is called as a loxodrome if the curve cuts all meridians at a constant angle on the tube with variable radius. 


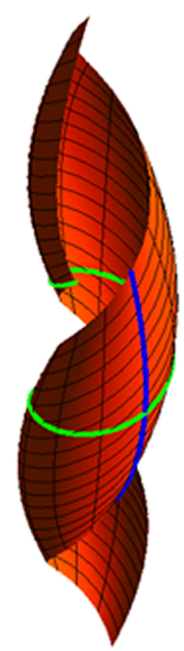

Figure 1. The projections of helicoidal surface, loxodrome (green), meridian (blue).

Let us consider the tube with variable radius $C$ which is given by Eq. (6). The tangent plane to $C$ at a point $p=C(u, v)$ is $\operatorname{span}\left\{C_{u}, C_{v}\right\}$.

A direct computation yields

$$
C_{u}=e_{1}(u)-r(u) \tau(u) \cos v e_{2}(u)+r^{\prime}(u) \cos v e_{3}(u)+r^{\prime}(u) \sin v e_{4}(u)
$$

and

$$
C_{v}=-r(u) \sin v e_{3}(u)+r(u) \cos v e_{4}(u) .
$$

By using these equations, the coefficients of first fundamental form of $C$ are

$$
E=1+r^{2}+r^{2} \tau^{2} \cos ^{2} v, F=0 \text { and } G=r^{2} .
$$

Assume that $E G-F^{2}=r^{2}+r^{2} r^{2}+r^{4} \tau^{2} \cos ^{2} v>0$, that is, $C$ is regular.

The first fundamental form of $C$ is given by

$$
d s^{2}=\left(1+r^{\prime 2}+r^{2} \tau^{2} \cos ^{2} v\right) d u^{2}+r^{2} d v^{2} .
$$

Also, the arc-length of any curve on $C$ between $u_{1}$ and $u_{2}$ is defined by

$$
s=\left|\int_{u_{1}}^{u_{2}} \sqrt{1+r^{\prime 2}+r^{2} \tau^{2} \cos ^{2} v+r^{2}\left(\frac{d v}{d u}\right)^{2}} d u\right| .
$$


Let us assume that $\sigma(t)$ is a curve on $C$. Then, we can write $\sigma(t)=C(u(t), v(t))$. With respect to the local base $\left\{C_{u}, C_{v}\right\}$, the vector $\sigma^{\prime}(t)$ has the coordinates $\left(u^{\prime}, v^{\prime}\right)$ and the vector $C_{u}$ has the coordinates $(1,0)$. At the point $p=C(u, v)$, where the loxodrome cuts the meridian at a constant angle $\varphi$, we get

$$
\cos \varphi=\frac{\left(1+r^{\prime 2}+r^{2} \tau^{2} \cos ^{2} v\right) d u}{\sqrt{\left(1+\left(r^{\prime 2}+r^{2} \tau^{2} \cos ^{2} v\right)^{2} d u^{2}+\left(1+r^{\prime 2}+r^{2} \tau^{2} \cos ^{2} v\right) r^{2} d v^{2}\right.}} .
$$

Then, differential equation of the loxodromes on the tubes with variable radius in $\mathbb{E}^{4}$ is given by

$$
\left(\frac{d v}{d u}\right)^{2}=\tan ^{2} \varphi\left(\frac{1+r^{2}}{r^{2}}+\tau^{2} \cos ^{2} v\right) .
$$

Furthermore, we put the condition that the second curvature of $\beta$ has null value. This means that $\beta$ is a 2-plane curve. Then, the general solution of Eq. becomes

$$
v=v(u)=\varepsilon \tan \varphi \int_{u_{0}}^{u} \frac{\sqrt{1+r^{\prime 2}}}{r} d u,
$$

where $\varepsilon$ is plus or minus.

Under this condition, we can give the following theorem.

Theorem 3. The loxodromes on the tubes with variable radius in $\mathbb{E}^{4}$ are

$$
\sigma(u)=\beta(u)+r(u)\left(e_{3}(u) \cos v(u)+e_{4}(u) \sin v(u)\right),
$$

where $v(u)=\varepsilon \tan \varphi \int_{u_{0}}^{u} \frac{\sqrt{1+r^{\prime 2}}}{r} d u$.

Now, we give the following example.

Example 2. Let us consider the base curve $\beta(u)=(u, 0,0,0)$. Taking $r(u)=$ $u, \varphi=\frac{\pi}{4}$ and $\varepsilon=1$, we have the following tube with variable radius:

$$
C(u, v)=(u, 0, u \cos v, u \sin v) .
$$

Using Eq. (21) and taking $u_{0}=1$, we get $v(u)=\sqrt{2} \ln |u|$. Taking $u \in(0.1,9)$, we have $v \in(-3.2563,3.1073)$. Then, the loxodrome is

$$
\sigma(u)=(u, 0, u \cos (\sqrt{2} \ln u), u \sin (\sqrt{2} \ln u)) .
$$

Also, by using Eq. (18), the arc-length of the loxodrome is approximately equal to 17.8.

Let us plot the graphs of the projections of tube with variable radius, loxodrome and meridian $(v=2)$ in $\mathbb{E}^{3}$ to see what they look like in $\mathbb{E}^{3}$ by using Mathematica plotting command

ParametricPlot3D $[\{x(u, v)+y(u, v), z(u, v), w(u, v)\},\{u, a, b\},\{v, c, d\}] ;$ 


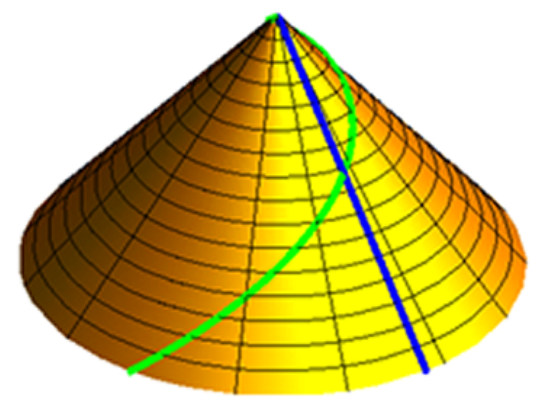

FiguRE 2. The projections of tubes with variable radius, loxodrome (green), meridian (blue).

\section{Conclusion}

Loxodromes are special curves which cut all meridians at a constant angle on the Earth's surface. They do not need a change of course. Thus, they are usually used in navigation. Loxodromes on rotational, helicoidal and canal surfaces in Euclidean 3 -space $\mathbb{E}^{3}$ were studied by different authors (see [11, [3], 4]). In this paper, we investigate the equations of loxodromes on helicoidal surfaces as well as the tubes with variable radius in Euclidean 4 -space $\mathbb{E}^{4}$, that is, we generalize the equations of loxodromes on helicoidal surfaces and canal surfaces in $\mathbb{E}^{3}$ to $\mathbb{E}^{4}$. The next time, we will investigate the differential equations of space-like and time-like loxodromes on the non-degenerate rotational surfaces, helicoidal surfaces and tubes with variable radius in Minkowski space-time $\mathbb{E}_{1}^{4}$.

\section{REFERENCES}

[1] Arslan, K., Bayram (Kılıç), B., Bulca, B., Kim, Y. H., Murathan, C., and Öztürk, G., Rotational embeddings in $\mathbb{E}^{4}$ with pointwise 1-type gauss map, Turk. J. Math., 35 (2011) 493-499.

[2] Arslan, K., Bayram (Kılıç), B., Bulca, B., and Öztürk, G., Generalized Rotation Surfaces in $\mathbb{E}^{4}$, Results. Math., 61 (2012) 315-327.

[3] Babaarslan, M., and Yayli, Y., Differential equation of the loxodrome on a helicoidal surface, Journal of Navigation, 68 (2015) 962-970.

[4] Babaarslan, M., Loxodromes on Canal Surfaces in Euclidean 3-Space, Ann. Sofia Univ. Fac. Math and Inf., 103 (2016) 97-103. 
[5] Bulca, B., Arslan, K., Bayram, B., and Öztürk, G., Canal surfaces in 4-dimensional Euclidean space, Int. J. Optim. Control, Theor. Appl. (IJOCTA), 7 (2017) 83-89.

[6] Dursun, U., and Turgay, N. C., General rotational surfaces in Euclidean space $\mathbb{E}^{4}$ with pointwise 1-type Gauss map, Math. Commun., 17 (2012) 71-81.

[7] Erdoğdu, M., and Özdemir, M., Generating Four Dimensional Rotation Matrices, doi: 10.13140/RG.2.1.4118.3442, 2015.

[8] Hieu D. T., and Thang, N. N., Bour's Theorem in 4-Dimensional Euclidean Space, Bull. Korean Math Soc., 54 (2017) 2081-2089.

[9] Gal, R. O., and Pal, L., Some notes on drawing twofolds in 4-dimensional Euclidean space, Acta Univ. Sapientiae, Informatica, 1(2) (2009) 125-134.

[10] Moore, C. L. E., Surfaces of Rotation in a Space of Four Dimensions, Ann. Math. (2), 21 (1919) 81-93.

[11] Noble, C. A., Note on loxodromes, Bull. Am. Math. Soc., 1-2 (1905) 116-119.

[12] Xu, Z., Feng, R., and Sun, J., Analytic and algebraic properties of canal surfaces, J. Comput. Appl. Math., 195 (2006) 220-228.

[13] Yoon, D. W., Rotation Surfaces with Finite Type Gauss Map in $\mathbb{E}^{4}$, Indian J. Pure Appl. Math., 32 (2001) 1803-1808.

[14] Yoon, D. W., Some Properties of the Clifford Torus as Rotational Surfaces, Indian J. Pure Appl. Math., 34 (2003) 907-915.

Current address, M. Babaarslan: Yozgat Bozok University, Department of Mathematics, 66100, Yozgat, Turkey

E-mail address: murat.babaarslan@bozok.edu.tr

ORCID Address: http://orcid.org/0000-0002-2770-4126 